\title{
Spontaneous urinoma debuting as retroperitoneal abscess: report of 2 cases and literature review
}

\author{
Alberto Artiles Medina, Inés Laso García, Gemma Duque Ruiz, Manuel Hevia Palacios, Fernando Arias \\ Funez, Francisco Javier Burgos Revilla
}

Department of Urology, Hospital Universitario Ramón y Cajal. Instituto Ramón y Cajal de Investigación Sanitaria (IRYCIS), Madrid, Spain

Correspondence to: Alberto Artiles Medina. Hospital Universitario Ramón y Cajal. M-607, km. 9, 100, 28034. Madrid, Spain.

Email: alberc.artiles@gmail.com.

\begin{abstract}
Spontaneous urinoma is a urological entity that can be complicated by a retroperitoneal abscess. Urinoma can be iatrogenic, traumatic, or can be caused by obstructive uropathy. We report two cases of spontaneous urinomas, describing a not previously published cause of urinoma: pyeloureteritis. (I) A 55-yearold Caucasian female started with intense left-sided flank pain for 3 days. CT scan revealed a voluminous retroperitoneal abscess, which extended through the posterior pararenal space and the left lumbar paravertebral musculature. (II) A 48-year-old Caucasian male presented with constitutional symptoms over the previous 2 months and investigations showed a voluminous urinoma and marked pyeloureteritis findings on CT scan. Both patients were managed by endoscopic placement of a double-J stent and drainage of the collection (open and percutaneous approach, respectively). Urinoma was confirmed by fluid biochemical analysis, which demonstrated that fluid creatinine was markedly raised as compared to the serum creatinine. Urinoma in the absence of obstruction or trauma is rare. These two case reports highlight one of the conditions leading to urinoma and the management of retroperitoneal abscess. Pyeloureteritis is a cause of spontaneous urinoma and it should be considered in the lack of another obstructive cause.
\end{abstract}

Keywords: Urinoma; spontaneous urinoma; retroperitoneal abscess; pyeloureteritis; case report

Submitted Aug 13, 2020. Accepted for publication Oct 28, 2020.

doi: $10.21037 /$ tau-20-1170

View this article at: http://dx.doi.org/10.21037/tau-20-1170

\section{Introduction}

A urinoma is defined as an encapsulated collection of urine outside the urinary tract as a result of disruption of the collecting system. It may be subcapsular in location, confined to the perirrenal space (extracapsular), or may transcend fascial boundaries and track to the peritoneal cavity. Quick diagnosis and adequate treatment of the underlying cause is crucial to prevent complications such as abscess formation and sepsis (1).

Rupture of the urinary tract may be post-traumatic, iatrogenic or a consequence of urinary tract obstruction. The term spontaneous implies that the rupture was not caused by iatrogenic manipulation, external trauma, prior surgery, degenerative kidney disease or external compression during intravenous urography. Obstructive uropathy may occur as a result of intrinsic or extrinsic obstruction of the urinary tract. Nevertheless, we did not identify any of the previously established causes (iatrogenic, traumatic or obstructive) of urinoma in these two cases.

Urinary leaks may be occult initially. The majority of urinomas reported in the literature have a delayed diagnosis because of the gradual and atypical onset of symptoms, including only malaise, vague loin or abdominal pain, weight loss, palpable mass and, when they become infected, sepsis (2). Sometimes, urine irritation in the peritoneal cavity can trigger inflammation leading to acute abdomen, so it can be misdiagnosed as appendicitis or diverticulitis (3).

Complications of urinomas include abscess formation and rupture. This complication is relatively rare (1) and when it occurs, the infection is generally produced by members of the Enterobacteriaceae group of bacteria. 
Table 1 Reported causes of urinoma

Trauma (penetrating or not injuries)

latrogenic (open surgery, laparoscopic, endoscopic or percutaneous procedures, including kidney biopsy)

Urolithiasis

Pregnancy and partum

Urothelial carcinoma (upper urinary tract or bladder)

Other malignancies

Retroperitoneal lymphadenopathy

Ureteropelvic Junction Obstruction

Urinary retention and elevated voiding pressure with detrusor sphincter dyssynergia

Extracorporeal Shock Wave Lithotripsy (ESWL)

Acute Interstitial Nephritis

Retroperitoneal fibrosis

Aortic aneurysm

Dermatomyositis

Posterior urethral valves

Pelvic abscess

Ureteric necrosis after kidney transplantation

Pyeloureteritis may simulate a urothelial neoplasm. Diagnosis is based on clinical symptoms and laboratory findings (4). Pyeloureteritis can potentially lead to ureterohydronephrosis and an increased intraluminal pressure in the ureter and renal pelvis. Inflammation (ureteritis, pyelonephritis) is included in the list of differential diagnosis for ureteral dilatation (5).

After ruling out the traumatic or iatrogenic cause and the presence of other causative conditions (Table 1), we hypothesize that the cause of these two cases is an inflammatory process of the urinary tract (pyeloureteritis). We present the following cases in accordance with the CARE reporting checklist (available at http://dx.doi. org/10.21037/tau-20-1170).

\section{Case presentation}

\section{Case 1}

A 55-year-old Caucasian female with history of Hashimoto's thyroiditis and major depressive disorder, started with intense left-sided flank pain 3 days prior to consultation. She denied fever, chills, nausea, vomiting, and hematuria. There had been no dysuria or urinary frequency. On questioning, the patient did not report trauma to the flank area. She had no history of urinary tract infection (UTI). The physical examination revealed the presence of a bulging, warm and tender area in the flank, without cellulitis.

The urinalysis test was consistent with massive pyuria. The renal function test was within the normal limits. The abnormal findings on laboratory testing were as follows: WBC count, $23.6 \times 10^{9} / \mathrm{L}$; neutrophil percentage, $84 \%$; C reactive protein: $286 \mathrm{mg} / \mathrm{L}$; procalcitonin, $166 \mathrm{ng} / \mathrm{mL}$.

The CT scan showed a voluminous abscess, which extended through the posterior pararenal space and the left lumbar paravertebral musculature (Figure 1). There were no stones located in the urinary tract.

It was initially managed with bilateral ureteral stent placement and percutaneous drainage of the perirenal left collection. A sample of the infected fluid was obtained and fluid biochemical analysis demonstrated fluid creatinine consistent with urinoma (fluid creatinine of $23 \mathrm{mg} / \mathrm{dL}$ ). The patient was given TZP (piperacillin, tazobactam). Urine and drainage fluid cultures revealed growth of Escherichia coli and the antibiogram guided the antimicrobial therapy. The antibiotic sensitivity revealed a high sensitivity to TZP and cephalosporins.

An abdominopelvic revaluation CT scan was performed on hospital day 7, revealing growth of total diameters of the retroperitoneal abscess. An open approach was used to drain the retroperitoneum through a Gibson incision because of the lack of improvement of the collection.

The patient's postoperative course was favourable and she was discharged on the $19^{\text {th }}$ hospital day. A repeat CT scan prior to discharge showed almost complete resolution of the abscess.

The double $\mathrm{J}$ catheter was removed on the $60^{\text {th }}$ postoperative day. The patient had remained asymptomatic all along. CT urography and urine cytology were performed for screening urothelial carcinoma and they were negative. There were no abnormal findings on follow-up CT urography and tests showed normal renal function.

\section{Case 2}

A 48-year-old Caucasian male with history of well-managed type 2 diabetes presented with constitutional symptoms over the previous 2 months and he was evaluated for unexplained weight loss, astenia and anorexia in the internal medicine department. Blood biochemistry tests indicated a serum 

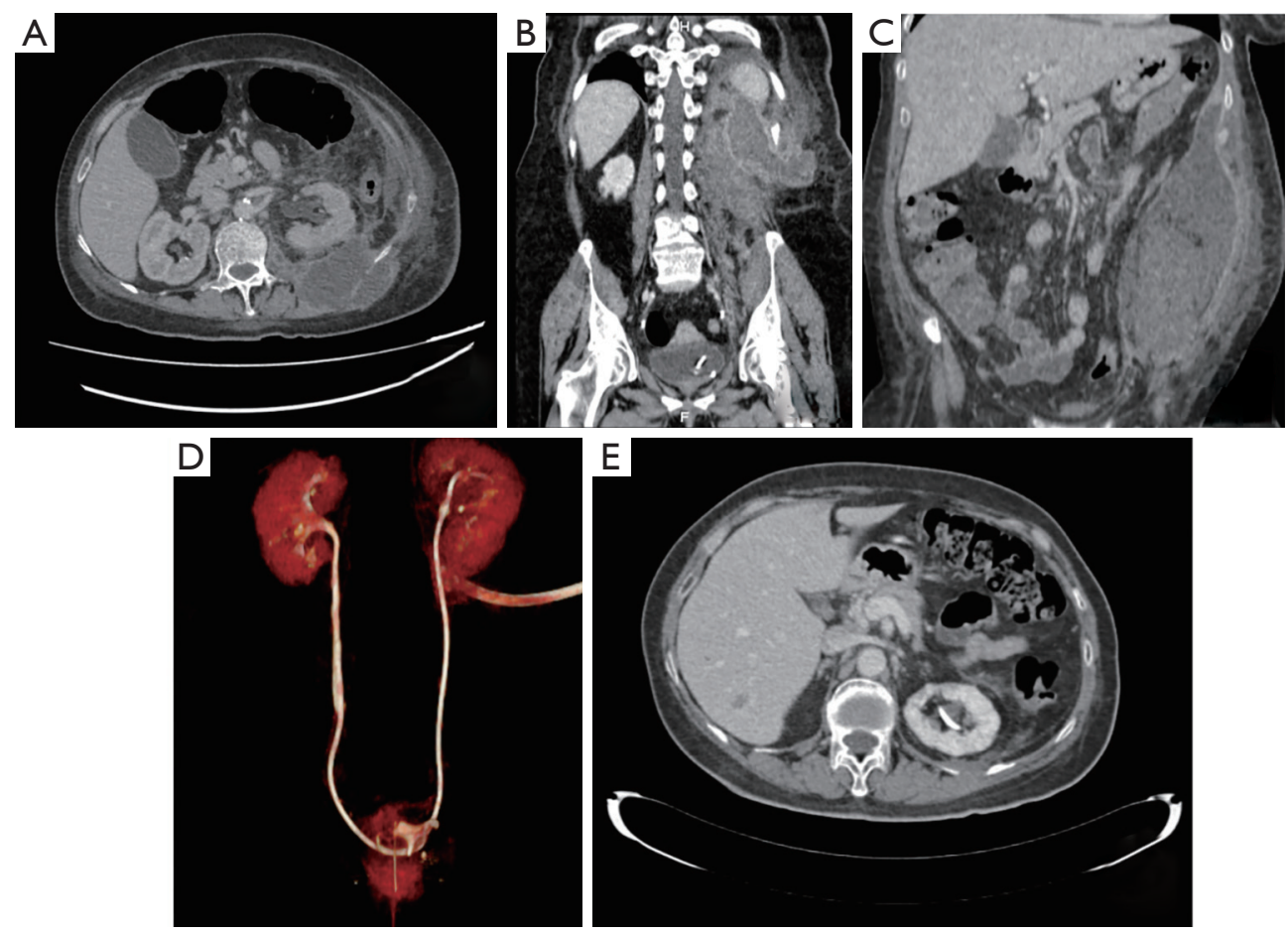

Figure $1 \mathrm{CT}$ scan shows a voluminous collection that extends through the posterior pararenal space and the left lumbar paravertebral musculature, and it comes close to posterior aspect of left kidney (A,B,C). (D) is a reconstruction image of properly placed drain tube. (E) shows an axial view of CT scan after treatment showing resolution of abscess.

creatinine level of $0.86 \mathrm{mg} / \mathrm{dL}$.

A CT urography demonstrated a fluid collection with peripheral rim enhancement measuring $8.6 \mathrm{~cm} \times 3.7 \mathrm{~cm} \times$ $4.6 \mathrm{~cm}$. It originated from perirenal space and spread along the anterior aspect of the psoas muscle. CT scan also showed thickening of the upper ureter and renal pelvis, probably due to inflammatory/infectious etiology (Figure 2).

A guided treatment with antibiotic (meropenem) was prescribed and a double $\mathrm{J}$ stent was inserted. Left retrograde pyelogram at the time of double $\mathrm{J}$ stent placement showed contrast extravasation, confirming a forniceal rupture, but there were no filling defects suggesting lithiasis, ureteral strictures or urothelial tumour. Furthermore, CT-guided percutaneous drainage was performed and analysis of the fluid revealed an increased creatinine level $(15.6 \mathrm{mg} / \mathrm{dL})$ indicating a urinary leak.

The next CT scan (10 days after) showed a collection reduced in size with resolution of the ring of enhancement. Two months later, the double $\mathrm{J}$ stent was removed, and a repeat CT urography revealed complete resolution of the collection and there were no filling defects. Urine cytology was used to rule out urothelial tumour. It was negative for malignant cells.

\section{Ethics statement}

All procedures performed in studies involving human participants were in accordance with the ethical standards of the institutional and/or national research committee(s) and with the Helsinki Declaration (as revised in 2013). Written informed consent was obtained from the patient for publication of this study and any accompanying images.

\section{Discussion}

Large spontaneous urinoma complicated by retroperitoneal abscess is a relatively rare urological entity, with only a small number of cases reported in the literature.

Spontaneous urinomas are usually the result of ureteral obstruction by calculus and the physiopathological mechanism underlying urinomas is the transmitted back pressure induced by blockage of the flow of urine (6). Other less common causes of urinoma are pelvic mass, pregnancy, retroperitoneal fibrosis, posterior urethral valves, or bladder 

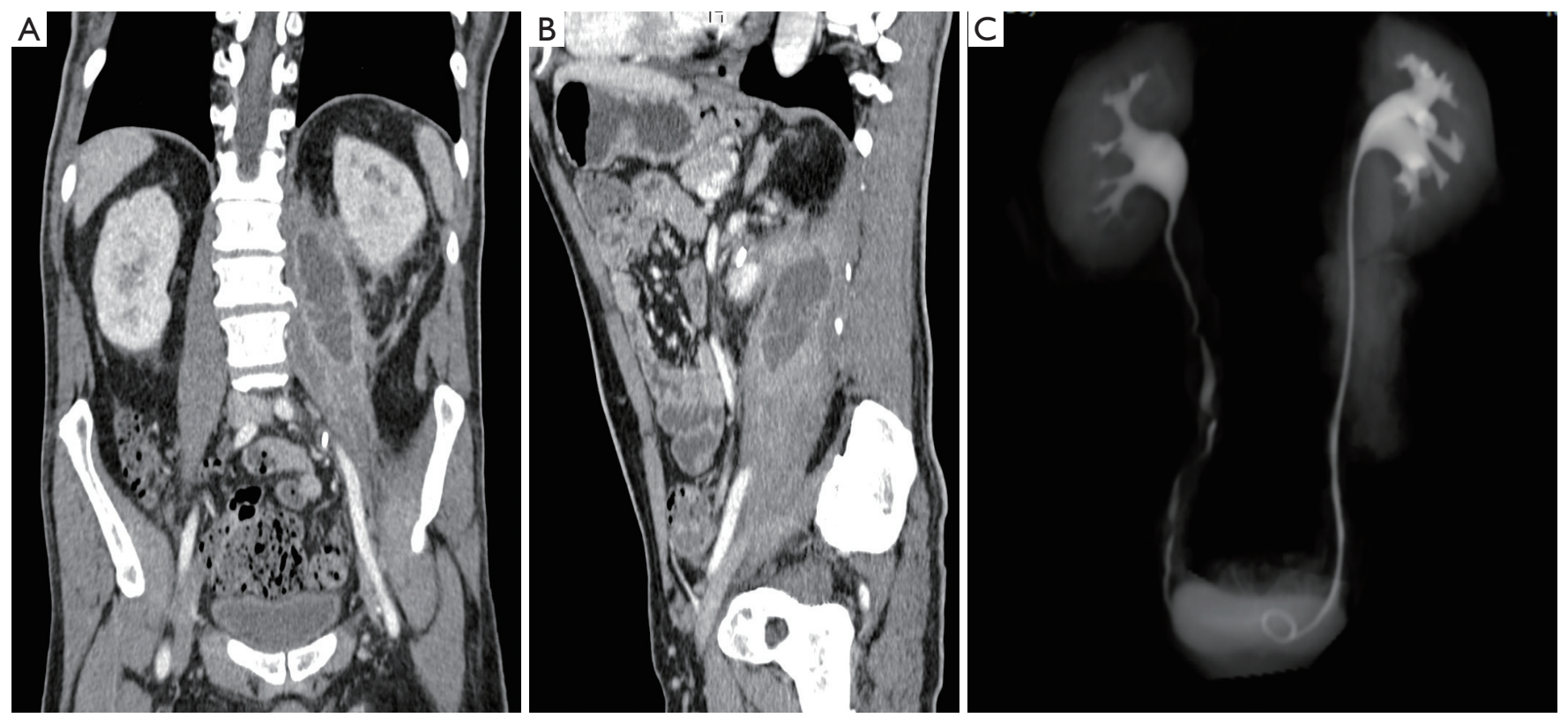

Figure 2 CT scan images (A,B) depicting a large collection with peripheral rim enhancement that is originating from perirenal space and spreading along the anterior aspect of the psoas muscle. The reconstruction image (C) illustrates the relationship between the collection and urinary tract.

outlet obstruction (7). Table 1 summarizes all the causes of spontaneous urinoma obtained from literature review. A Pubmed search was conducted in order to identify causes of spontaneous urinoma. Search terms were "urinoma", "urinary leak", "ureteral rupture" or "renal pelvis rupture" in title and abstract.

Forniceal rupture secondary to iatrogenic (including due to invassive endourological procedure) and traumatic (blunt or penetrating) mechanism are the most frequent causes of urinomas. When urinomas are not traumatic or iatrogenic, the likely cause varies with the age of the patient. In neonates posterior urethral valves are a common cause and in older children a congenital obstruction (such as ureteropelvic obstruction) would be more likely than obstruction due to ureteral calculi or malignancy, which is more frequent in adults (8). In our cases, a spontaneous rupture of the urinary tract in the context of an inflammation of the urinary tract was the origin of the infected urinoma. Spontaneous stone expulsion causing urinoma was excluded, because there was no evidence of stones in CT imaging (including bladder stone), the patients had no personal or familiar history of calculi, and finally they denied stone expelling or hematuria suggesting renal colic.

Spinelli et al. reported a series of 31 patients with upper urinary tract rupture admitted to the Emergency
Department for renal colic. Out of 1,629 patients diagnosed with renal colic, upper urinary tract rupture (categorized in 3 stages: local spread, free fluid and urinoma) was only reported in $1.9 \%$. In this series only 2 urinomas were observed. Most of the spontaneous upper urinary tract ruptures appeared to result from the local spread of urine deriving from a fornix rupture (54.84\%) (9).

Urinary tract infections (UTIs) represent the most common urologic disease and occasionally they can ascend from the lower urinary tract and may reach the renal pelvis and involve the kidney by tubulo-interstitial inflammation resulting in acute pyelonephritis. In some cases, pelvicalyceal and/or ureteral-circumferential mural thickening and diffuse hyperenhancement corresponding to urothelial inflammation may be the only imaging feature suggesting the presence of an ongoing ascending UTI on CT scan. Differential diagnosis includes primary urothelial malignancies, ureteritis, tuberculosis, and other uncommon ureteral disorders such as ureteral amyloidosis, metastases, haematomas, lymphoma, and retroperitoneal fibrosis (10). Urinoma associated to these urinary tract inflammatory changes can be explained by the obstructive effect of the inflammation, excluding other recognised causes of urinary tract obstruction.

In 1976, Kass et al. reported non-obstructive hydronephrosis 
as a urographic finding in acute pyelonephritis (11). In consonance with that author, Rathi et al. conducted a study to assess the significance of ureteral dilatation with no obvious cause on intravenous urography in 18 patients. In 8 out of these 18 cases, it was associated with urinary tract infection (pyuria on urine microscopic examination: two; E. coli infection on urine culture: three; ureteritis on CT urography: three) (12).

There is no solid consensus about the ideal management of urinoma and guidelines of the major urological societies worldwide do not include urinoma in the management algorithm as a complication of urolithiasis (13). Small urinomas may resorb spontaneously and, therefore, conservative management can be chosen. Nevertheless, large urinomas - even without systemic signs-usually need intervention, either in the form of percutaneous drainage (a drainage catheter can be placed with ultrasound or CT guidance), ureteral stenting (endourological treatment), or open surgery (as exemplified by the case 1) (7). Fluid culture is recommended to guide antibiotic treatment (14). In the series of Spinelli et al., spontaneous upper urinary tract rupture was actively treated in $30 / 31$ patients: 26 ureteric stent, 1 nephrostomy and 3 primary ureteroscopic lithotripsy followed by double-J stenting. All the patients underwent an intraoperative retrograde pyelography, confirming extravasation in only 9 cases (30\%) (9).

In conclusion, urinoma in the absence of obstruction or trauma is rare. These case reports highlight one of the variety of conditions leading to urinomas and the management of superinfected urinoma, that represents a severe complication of this entity. The contribution of this paper is to add a new differential diagnosis to the list of causes of urinoma: an inflammatory urinary tract process (pyeloureteritis).

\section{Acknowledgments}

Funding: None.

\section{Footnote}

Reporting Checklist: The authors have completed the CARE reporting checklist. Available at http://dx.doi.org/10.21037/ tau-20-1170

Conflicts of Interest: All authors have completed the ICMJE uniform disclosure form (available at http://dx.doi. org/10.21037/tau-20-1170). The authors have no conflicts of interest to declare.

Ethical Statement: The authors are accountable for all aspects of the work in ensuring that questions related to the accuracy or integrity of any part of the work are appropriately investigated and resolved. All procedures performed in studies involving human participants were in accordance with the ethical standards of the institutional and/or national research committee(s) and with the Helsinki Declaration (as revised in 2013). Written informed consent was obtained from the patient for publication of this study and any accompanying images.

Open Access Statement: This is an Open Access article distributed in accordance with the Creative Commons Attribution-NonCommercial-NoDerivs 4.0 International License (CC BY-NC-ND 4.0), which permits the noncommercial replication and distribution of the article with the strict proviso that no changes or edits are made and the original work is properly cited (including links to both the formal publication through the relevant DOI and the license). See: https://creativecommons.org/licenses/by-nc-nd/4.0/.

\section{References}

1. Vaidya R, Swetz KM. Urinoma presenting as an abscess in an immunocompromised host: A case report. J Med Case Rep 2013;7:193.

2. Christodoulidou M, Clarke L, Napier-Hemy RD. Infected urinoma secondary to a ruptured renal calyx from a partial staghorn stone. J Surg Case Rep 2015;2015:rjv096.

3. Pace K, Spiteri K, German K. Spontaneous Proximal Ureteric Rupture Secondary to Ureterolithiasis. J Surg Case Rep 2017;2016:rjw192.

4. Coll DG, Yanguas C, Diaz-Ruiz MJ, et al. Thickening of the renal pelvis: spectrum of CT findings - EPOSTM. ECR 2014. doi: 10.1594/ecr2014/C-1708.

5. Ureteritis - an overview I ScienceDirect Topics [Internet]. [cited 2020 May 23]. Available online: https:// www.sciencedirect.com/topics/veterinary-science-andveterinary-medicine/ureteritis

6. Kontaki T, Drevelegkas K, Markou A, et al. Spontaneous urinomas as a complication of ureterolithiasis: MDCT findings. ECR 2012. doi: 10.1594/ecr2012/C-0555.

7. Pyrpasopoulou A, Georgopoulou V, Anyfanti P, et al. Spontaneous urinoma: An unexpected cause of acute abdomen. Am J Emerg Med 2011;29:695.e3-695.e4.

8. Lee J, Darcy M. Renal cysts and urinomas. Semin 
Intervent Radiol 2011;28:380-91.

9. Spinelli MG, Palmisano F, Zanetti SP, et al. Spontaneous upper urinary tract rupture caused by ureteric stones: A prospective high-volume single centre observational study and proposed management. Arch Esp Urol 2019;72:590-5.

10. Massimo T. Acute pyelo-ureteritis: MDCT diagnosis and follow-up. Eurorad 2013. DOI: 10.1594/EURORAD/ CASE.10764.

11. Kass EJ, Silver TM, Konnak JW, et al. The Urographic Findings in Acute Pyelonephritis: Non-Obstructive Hydronephrosis. J Urol 1976;116:544-6.

Cite this article as: Artiles Medina A, Laso García I, Duque Ruiz G, Hevia Palacios M, Arias Funez F, Burgos Revilla FJ. Spontaneous urinoma debuting as retroperitoneal abscess: report of 2 cases and literature review. Transl Androl Urol 2021;10(2):963-968. doi: 10.21037/tau-20-1170
12. Rathi V, Agrawal S, Bhatt S, et al. Ureteral Dilatation with No Apparent Cause on Intravenous Urography: Normal or Abnormal? A Pilot Study. Adv Urol 2015;2015:681836.

13. Al-Mujalhem AG, Aziz MS, Sultan MF, et al. Spontaneous forniceal rupture: Can it be treated conservatively? Urol Ann 2017;9:41-4.

14. Goldwasser J, Wahdat R, Espinosa J, et al. Urinoma: Prompt Diagnosis and Treatment Can Prevent Abscess Formation, Hydronephrosis, and a Progressive Loss of Renal Function. Case Rep Emerg Med 2018;2018:5456738. 